\title{
ACIDENTE VASCULAR CEREBRAL: ACHADOS CLÍNICOS E PRINCIPAIS COMPLICAÇÕES
}

\author{
CEREBROVASCULAR ACCIDENT: CLINICAL FINDINGS AND MAJOR COMPLICATIONS
}

\author{
Lais Samara Melo ${ }^{\mathrm{a}^{*}}$, Livia Maria Souza Emerick ${ }^{\mathrm{b}^{*}}$, Patrícia Natalia Medeiros Alves ${ }^{\mathrm{c}^{*}}$, Thais Batista \\ Rocha $^{\mathrm{d}^{*}}$, Vania Regina Goveia ${ }^{\mathrm{e} * *}$, Gilberto Lima Guimarães ${ }^{\mathrm{f}^{* *}}$, Isabel Yovana Mendoza ${ }^{* *}$ \\ alaisdtna@yahoo.com.br, ${ }^{b}$ liviamaria_2013@yahoo.com.br, ${ }^{c}$ patricianm.alves@yahoo.com.br, ${ }^{d}$ thays.rocha@hrtn.fundep.ufmg.br, ${ }^{\mathrm{e} v a n i a g o v-}$ \\ eia@ufmg.br, fdrgilberto.guimaraes@hotmail.com, gisabelyovana@ufmg.br \\ *Hospital Risoleta Tolentino Neves - Belo Horizonte (MG), Brasil \\ **Universidade Federal de Minas Gerais - Belo Horizonte (MG), Brasil
}

Data de recebimento do artigo: 03/11/2015 Data de aceite do artigo: 25/02/2016

\section{RESUMO}

Introduçáo: $\mathrm{O}$ acidente vascular cerebral (AVC) é uma das mais importantes causas de mortes no mundo, provocado pela obstrução ou rompimento de uma ou mais artérias, gerando falta de circulação sanguínea no cérebro. Objetivo: Verificar as condiçóes clínicas dos pacientes e as complicações decorrentes do AVC em pacientes hospitalizados. Método: Estudo retrospectivo, exploratório, realizado por meio da análise de prontuários dos pacientes admitidos na unidade de internação, com o diagnóstico clínico de AVC isquêmico ou hemorrágico, no período de $1^{\circ}$ de janeiro a 31 de dezembro de $2011 \mathrm{em}$ um hospital universitário em Belo Horizonte/MG. A amostra foi composta por 314 prontuários de pacientes. Resultados: Dentre os pacientes vítimas de AVC, 94\% apresentaram AVC isquêmico. Foram identificados os fatores de risco: hipertensão arterial sistêmica (HAS) em 74\% dos pacientes, diabetes mellitus em $25 \%$ e tabagismo em $22 \%$. A média de tempo de internação foi de 11 dias. Complicaçóes associadas ao uso de dispositivos invasivos foram apresentadas por $23 \%$ dos pacientes. Dentre as principais complicaçôes estão: infecção do trato urinário, úlceras por pressão e pneumonia. Conclusão: Evidencia-se que a idade, o tempo de internaçáo e a presença de dispositivos invasivos interferem na ocorrência de complicaçóes, sendo necessário diminuir o tempo de internaçáo e estabelecer critérios para o uso de dispositivos invasivos.

Palavras-chave: Acidente vascular cerebral; complicaçōes; enfermagem; idoso.

\section{ABSTRACT}

Introduction: cerebrovascular accident (CVA) is one of the leading causes of deaths worldwide caused by blockage or rupture of one or more arteries, causing a lack of blood circulation in the brain. Objective: To verify patients clinical conditions and complications of stroke in hospitalized patients. Method: a retrospective, exploratory study, analysing medical records of patients admitted to the inpatient unit, with a clinical diagnosis of ischemic or hemorrhagic stroke, for the period January-December 2011, in a university hospital in Belo Horizonte, state of Minas Gerais. The sample consisted of 314 patients' medical records . Results: Among the patients that suffered a stroke, 94\% had ischemic stroke. The risk factors were identified as: systemic arterial hypertension in $74 \%$ of patients, DM in $25 \%$ and smoking in $22 \%$. The average hospitalization time was 11 days and $23 \%$ of patients had complications associated with the use of invasive devices. The major complications are: urinary tract infection, pressure ulcers and pneumonia. Conclusion: it has become evident that the age, length of stay and the presence of invasive devices are associated with the occurrence of complications, being necessary to decrease length of stay and establish criteria for the use of invasive devices.

Keywords: Cerebrovascular accident; complications; nursing; elderly. 


\section{Introdução}

Uma das mais importantes causas de mortes no mundo, o acidente vascular cerebral (AVC) trata-se de uma síndrome caracterizada pelo início agudo de um déficit neurológico que persiste por pelo menos 24 horas, e é resultado de um distúrbio na circulação cerebral ${ }^{1}$.

Popularmente conhecido como derrame, a doença atinge 16 milhóes de pessoas no mundo a cada ano ${ }^{2}$. No Brasil, ela representa a primeira causa de morte e incapacidade, gerando grande impacto econômico e social. De acordo com dados do Ministério da Saúde, em 2011 foram registrados 99.159 óbitos por AVC2.

O AVC é classificado em dois grandes grupos: AVC isquêmico o AVC hemorrágico. $\mathrm{O}$ mais frequente, com cerca de $90 \%$ dos casos, é o AVC isquêmico, que se caracteriza pela interrupção do fluxo sanguíneo em uma determinada área do encéfalo, podendo acarretar distúrbios de linguagem, perda do equilíbrio ou coordenação, distúrbios visuais, bem como a perda do controle dos esfíncteres anal e vesical ${ }^{3,4}$. O AVC hemorrágico compreende a hemorragia subaracnóidea, em geral decorrente da ruptura de aneurismas saculares congênitos localizados nas artérias do polígono de Willis e a hemorragia intraparenquimatosa, cujo mecanismo causal básico é a degeneração hialina de artérias intraparenquimatosas cerebrais, tendo como principal doença associada à hipertensão arterial sistêmica (HAS) ${ }^{4}$.

Sabe-se que os déficits neurológicos decorrentes do AVC variam conforme a localização da lesão vascular, do tempo de perfusáo inadequada e da existência de circulação colateral ${ }^{4}$. Nesse sentido, para minimizar os déficits potenciais causados pelo AVC, o cuidado na fase aguda deve ser oportuno no tempo e efetivo para impedir a morte do tecido cerebral. Dessa forma, com o esperado aumento na incidência de AVC no Brasil, nas próximas décadas, estes resultados reforçam a necessidade de cuidados agudos e preventivos eficazes ${ }^{5}$.

No entanto, além dos riscos inerentes à fase aguda, o processo de internação também pode contribuir significativamente para a morbimortalidade por essa doença. Complicações clínicas, neurológicas e psiquiátricas interferem na recuperação e aumentam o custo do tratamento ${ }^{6}$.

Complicações, tais como pneumonia e infecção do trato urinário (ITU) dentre outras, foram associadas a um maior tempo de internaçáo, morbidade e morte dos pacientes. Entretanto, as complicaçóes podem ser minimizadas através da atuação de todos os profissionais envolvidos no processo. Medidas de prevenção e reabilitação precoce podem melhorar os prognósticos após o AVC, reduzir o risco de complicaçóes e consequentemente diminuir a morbimortalidade ${ }^{6}$.
Nesse contexto, questionam-se: Quais os fatores associados à presença de complicaçóes e qual o tempo de internação desses pacientes em uma unidade de referência para AVC?

Sendo assim, este estudo tem por objetivo geral verificar as condiçóes clínicas dos pacientes e as complicaçóes decorrentes do AVC em pacientes hospitalizados; e por objetivos específicos identificar as características demográficas, sociais e fatores de risco relacionados ao AVC; conhecer o período de internação, complicaçôes e o uso de dispositivos invasivos durante a hospitalização.

Este estudo torna-se relevante devido à necessidade premente de rever os fluxos e práticas em saúde relacionadas aos pacientes vítimas de AVC, tendo em vista as repercussões na morbimortalidade e qualidade de vida deles. Espera-se que este trabalho ajude na compreensão das medidas que envolvem a prevenção de complicaçóes, a fim de permitir aos agentes envolvidos propor intervençôes mais resolutivas e impactantes.

\section{Metodologia}

Trata-se de um estudo retrospectivo exploratório, realizado em uma unidade de AVC de um hospital campo de estudo na cidade de Belo Horizonte/MG. A amostra deste estudo foi, inicialmente, constituída por 343 prontuários de pacientes admitidos nessa unidade, com o diagnóstico clínico de AVC isquêmico ou hemorrágico, no período de $1^{\circ}$ de janeiro a 31 de dezembro de 2011. A lista com o nome dos pacientes foi cedida pelo setor de Tecnologia de Informação do referido hospital. No entanto, durante a coleta de dados foram excluídos 29 prontuários de pacientes por terem diagnóstico clínico diferente de AVC isquêmico ou hemorrágico ou por terem sido admitidos em um período diferente do proposto pelo estudo. Sendo assim, a amostra do estudo constituiu-se de 314 pacientes.

A coleta de dados se deu por meio de consulta ao prontuário eletrônico dos pacientes, sendo analisadas as evoluçôes médicas e de enfermagem, no próprio hospital. Os dados coletados foram registrados em um impresso próprio elaborado pelas autoras.

Os dados foram armazenados no programa Microsoft Excel 2010 e processados no programa Statistical Package for Social Sciences (SPSS, versão 15.0 para Windows). Dois tipos de análise foram realizados: análise descritiva, na qual foram registrados os valores de frequência absoluta e relativa, média e desvio-padrão das variáveis sociodemográficas e clínicas dos pacientes. Para verificar a associação da variável dependente "Complicaçóes" ( $0=$ sem complicaçóes 
e 1 = com complicaçóes) com as variáveis independentes: sexo, idade, fatores de risco para AVC, tempo de internação, dispositivos invasivos ou número de dispositivos invasivos foi utilizada a análise de regressão logística múltipla, baseada no método forward stepwise (likelihood ratio). A escolha das variáveis para inclusão no modelo foi baseada em fundamentação teórica e, somente aquelas que, na análise bivariada, apresentavam $\mathrm{p}<0,10$ foram incluídas. Após os ajustes, permaneceram no modelo as variáveis: idade, tempo de internação e número de dispositivos invasivos. A análise de ajuste do modelo logístico final foi realizada pelo teste de Hosmer-Lemeshow e pela análise dos resíduos. A força de associação de cada variável independente com as "complicaçôes" foi expressa em odds ratio (OR) com intervalo de confiança de 95\% (IC 95\%) e o nível de significância adotado foi $\mathrm{p}<0,05$.

Para a realização desta pesquisa foi considerada a normatização prevista na Resolução 446/12 do Conselho Nacional de Saúde que regulamenta a pesquisa com seres humanos. A coleta de dados ocorreu após a aprovação do projeto pelo Núcleo de Ensino e Pesquisa (NEPE) do hospital campo do estudo e pelo Comitê de Ética em Pesquisa (COEP) da Universidade Federal de Minas Gerais (CAE: 04987712.7.0000.5149).

\section{Resultados}

$\mathrm{Na}$ caracterização da população, em relação ao sexo foi possível perceber que houve maior acometimento de homens $164(52 \%)$ se comparado ao número de mulheres 150 (48\%); a faixa etária mais acometida foi de 70 a 79 anos com 86 (27\%), seguida de 60 a 69 anos com 69 (22\%) (Tabela 1).

Em relação ao tipo de AVC sofrido, os dados apontam grande predomínio do AVC isquêmico sobre o AVC hemorrágico. Vale ressaltar que o AVC hemorrágico a que estamos nos referindo é o $\mathrm{AVC}$ hemorrágico intraparenquimatoso, uma vez que os pacientes com hemorragia subaracnóidea não são encaminhados para a unidade de AVC, e sim para outros setores na instituição.

Outro fator que interfere diretamente no acometimento de AVC são os fatores de risco a que estes pacientes estão expostos. Dos 314 prontuários de pacientes avaliados, $34(11 \%)$ não apresentavam os fatores de risco pesquisados, sendo que os outros 280 (89\%) pacientes possuíam pelo menos um dos fatores de risco apresentados na Tabela 1 . Entre eles, destacam-se a HAS (74\%), o diabetes mellitus (25\%) e o tabagismo (22\%), como sendo os mais prevalentes nessa população.

A maior parte dos pacientes permaneceu na unidade de 5 a 32 dias, sendo a média do tempo de internação de 11 dias $(\mathrm{dp} \pm 7,8)$. No entanto, embora náo seja o objetivo deste estudo, vale ressaltar que antes de serem admitidos na unidade de AVC, esses pacientes ficam em observação no Pronto Atendimento (PA), aguardando vaga na unidade. No PA a média de permanência desses pacientes é de três dias (Tabela 1).

Em relação ao uso de dispositivos invasivos, 83\% dos pacientes utilizaram pelo menos um dos dispositivos pesquisados.

Tabela 1: Condições clínicas dos pacientes vítimas de AVC internados em uma unidade de AVC. Belo Horizonte, 2012.

\begin{tabular}{|c|c|c|c|}
\hline Variável & $\mathbf{N}$ & $\%$ & Média (dp) \\
\hline \multicolumn{4}{|l|}{ Tipo de AVC* } \\
\hline Isquêmico & 294 & 94 & \\
\hline $\begin{array}{l}\text { Hemorrágico } \\
\text { intraparenquimatoso }\end{array}$ & 20 & 06 & \\
\hline \multicolumn{4}{|l|}{ Fatores de risco para AVC } \\
\hline $\mathrm{HAS}^{\dagger}$ & 231 & 74 & \\
\hline Diabetes mellitus & 77 & 25 & \\
\hline Tabagismo & 68 & 22 & \\
\hline AVC prévio & 59 & 19 & \\
\hline Cardiopatias & 50 & 15 & \\
\hline Arritmia & 37 & 12 & \\
\hline Dislipidemia & 32 & 11 & \\
\hline Etilismo & 31 & 10 & \\
\hline Epilepsia & 8 & 3 & \\
\hline Doença de Chagas & 7 & 2 & \\
\hline Obesidade & 6 & 2 & \\
\hline Outros & 9 & 9 & \\
\hline \multicolumn{4}{|c|}{ Tempo de internaçáo (dias) } \\
\hline $0-4$ & 35 & 11 & \\
\hline $5-9$ & 102 & 33 & \\
\hline $10-14$ & 72 & 23 & \\
\hline $15-19$ & 39 & 12 & $11(7,8)$ \\
\hline $20-24$ & 31 & 10 & \\
\hline $25-29$ & 14 & 4 & \\
\hline$\geq 30$ & 21 & 7 & \\
\hline \multicolumn{4}{|l|}{ Dispositivos invasivos } \\
\hline Acesso venoso periférico & 243 & 77 & \\
\hline Acesso venoso central & 4 & 1 & \\
\hline Sonda vesical de demora & 11 & 3 & \\
\hline Sonda vesical de alívio & 15 & 5 & \\
\hline Sonda nasoentérica & 55 & 18 & \\
\hline Nenhum & 54 & 17 & \\
\hline
\end{tabular}


Tabela 2: Complicações dos pacientes vítimas de AVC em relação ao número total de pacientes internados em uma unidade de AVC. Belo Horizonte, 2012.

\begin{tabular}{|c|c|c|}
\hline Complicaçóes & $\mathbf{n}$ & $\%$ \\
\hline ITU* & 28 & 8,9 \\
\hline Úlceras por pressão (estágio II e III) & 27 & 8,5 \\
\hline Pneumonia & 25 & 7,9 \\
\hline Transformação hemorrágica & 8 & 2,5 \\
\hline Insuficiência renal aguda & 6 & 1,9 \\
\hline Flebite & 4 & 1,2 \\
\hline Trombose venosa profunda & 3 & 0,9 \\
\hline Bacteremia & 1 & 0,3 \\
\hline Hemorragia digestiva baixa & 1 & 0,3 \\
\hline Sepse & 1 & 0,3 \\
\hline
\end{tabular}

Fonte: Dados da pesquisa.

*ITU: infecção do trato urinário.

Dos 314 prontuários de pacientes avaliados, aproximadamente $23 \%$ dos pacientes apresentaram algum tipo de complicação. As mais prevalentes foram: ITU $(8,9 \%)$, úlceras por pressão $(8,5 \%)$ e pneumonia $(7,9 \%)$. Também foram encontradas complicaçóes como transformação hemorrágica $(2,5 \%)$, insuficiência renal aguda $(1,9 \%)$, flebite $(1,2 \%)$, trombose venosa profunda $(0,9 \%)$, bacteremia $(0,3 \%)$, hemorragia digestiva baixa $(0,3 \%)$ e sepse $(0,3 \%)$ (Tabela 2$)$.

Devido à subnotificação das úlceras por pressão estágio I (hiperemia não reativa), optou-se por não coletar também essa informação. Grande parte dos pacientes (77\%) não apresentou nenhum tipo de complicação durante a internação na unidade de AVC, descrita em prontuário.

De acordo com os resultados do modelo de regressão logística apresentada na Tabela 3, as variáveis que se associaram significativamente com a presença de complicações foram: idade, tempo de internação e uso de dispositivos invasivos.

Tabela 3: Variáveis associadas à presença de complicações em pacientes vítimas de AVC, internados em uma unidade de AVC. Belo Horizonte, 2012.

\begin{tabular}{cccccccc} 
Variáveis & B & Wald & $\begin{array}{c}\text { Valor } \\
\text { (SE) }\end{array}$ & & de p & OR & $\begin{array}{c}\text { 95\% C.I. for } \\
\text { EXP(B) }\end{array}$ \\
Idade & 0,109 & 19,430 & 0,000 & 1,115 & 1,063 & 1,171 \\
\hline $\begin{array}{c}\text { Tempo de } \\
\text { internaçáo }\end{array}$ & 0,158 & 25,243 & 0,000 & 1,171 & 1,101 & 1,245 \\
$\begin{array}{c}\text { Dispositivos } \\
\text { invasivos }\end{array}$ & 1,765 & 21,275 & 0,000 & 5,840 & 2,759 & 12,362 \\
\hline
\end{tabular}

Fonte: Dados da pesquisa.

$\mathrm{B}$ (SE): coeficiente de regressão e erro-padrão; Wald: estatística de Wald; OR: odds ratio; $\operatorname{Exp}(\mathrm{B})$ : odds ratio - OR; IC 95\%: intervalo de confiança de $95 \%$.
O uso de dispositivos invasivos aumentou em 5,8 vezes (IC 95\%: 2,76-12,362) o risco de complicaçóes, seguido pelo tempo de internação (OR: 1,17) e pela idade (OR: 1,12). Neste modelo, foram excluídos 25 pacientes considerados "outliers", com capacidade de predição de 90,7\%.

\section{Discussão}

Em relaçáo ao sexo, os resultados do estudo mostram um maior número de homens sendo acometidos por AVC, se comparado ao número de mulheres. Este fato corrobora os dados encontrados em outro estudo, inclusive na mesma proporçãa ${ }^{7}$.

Relatos da literatura sugerem que os homens têm dificuldade em reconhecer suas necessidades e rejeitam a possibilidade de adoecer, devido à cultura patriarcal. Isso poderia ser apontado como um dos fatores que favorece o adoecimento deles e contribui para os altos índices de morbimortalidade nessa populaçãa ${ }^{8}$. No entanto, por se tratar de uma doença que acomete principalmente pacientes idosos, existe uma tendência para que cada vez mais mulheres sejam acometidas por AVC, visto que a expectativa de vida dos brasileiros tem aumentado a passos largos e a feminização da velhice é uma realidade, o que faz este grupo estar mais vulnerável aos problemas de saúde?

No que diz respeito à idade, vários estudos também demonstram que o AVC é predominante na faixa etária de 70 a 79 anos $^{7,10}$. Sabe-se que esse evento pode ocorrer em qualquer faixa etária, entretanto, sua incidência aumenta com o passar dos anos, dobrando a cada década de vida, após os 55 anos de vida ${ }^{11}$.

$\mathrm{Na}$ população estudada, foi possível identificar o predomínio de pacientes vítimas de AVC isquêmico sobre o AVC hemorrágico, o que condiz com dados da literatura, uma vez que mais de $85 \%$ dos AVC's são de etiologia isquêmica ${ }^{2}$. Neste estudo, encontrou-se uma desproporçáo, que pode ser atribuída ao fato de que os casos de AVC hemorrágicos intraparenquimatosos graves e subaracnóideos são, na maioria das vezes, encaminhados diretamente para o Centro de Terapia Intensiva (CTI), não sendo dessa forma, internados na unidade de AVC.

Em relação aos fatores de risco, a HAS, o diabetes mellitus, o tabagismo e as arritmias, especialmente a fibrilação atrial, são considerados os principais fatores que predispóem ao acometimento por $\mathrm{AVC}^{6}$. Dentro dos fatores de risco pesquisados neste estudo, apenas uma pequena parcela dessa população não possui nenhum fator de risco associado. Por se tratar de uma doença multifatorial, os fatores de risco acumulados ao longo da vida podem desencadear a ocorrência do AVC. Sendo assim, identificar e registrar corretamente em prontuários os fatores de risco a que esses pacientes 
estão expostos é extremamente importante, pois, reflete o perfil dessa população e auxilia os profissionais da saúde na intervenção de forma eficaz e na elaboração de um plano de alta consistente, buscando reduzir a reincidência desse agravo da saúde.

Em relação ao tempo de internaçáo, em geral as unidades de AVC integrais no país têm tempo de internação em torno de 15 dias. Esse tempo de internação foi determinado a título de financiamento de leitos. No entanto, dentro do conceito de unidades de AVC integrais estas devem dar continuidade ao tratamento da fase aguda, reabilitação precoce e investigação etiológica completa, podendo o paciente necessitar extrapolar esse tempo de internação.

Dessa forma, percebe-se que a média do tempo de internação dos pacientes na unidade de AVC é compatível com uma unidade de AVC descrito pela literatura. No entanto, ao considerar o tempo de internação na instituição (incluindo o tempo de internação em observação no PA), percebe-se que este está no limite. É válido ressaltar que existe a preocupação por parte da equipe para que o paciente esteja estável para ser contrarreferenciado à rede de atenção básica, fato que pode estar influenciando no tempo de internação.

Dentre as complicaçóes encontradas nos pacientes pós-AVC agudo encontra-se as ITU e pneumonias. Um estudo de meta-análise ${ }^{12}$ que incluiu 87 estudos, envolvendo 137.817 pacientes pós-AVC, evidenciou a presença de $10 \%$ de ITU e $10 \%$ de pneumonia nos estudos analisados. Percebem-se neste estudo taxas inferiores dessas complicaçóes (8,9\% ITU e 7,9\% pneumonia). No entanto, vale ressaltar que no estudo citado os autores incluíram estudos realizados em Unidades de AVC, Unidades Clínicas e Unidades de Terapia Intensiva (UTI). Quando analisados apenas os estudos com pacientes internados em outras unidades não UTI, as taxas de ITU são de $10 \%$ e de pneumonia são de $9 \%$.

Sabe-se que o desenvolvimento dessas infecçóes pós-AVC dependem de múltiplos fatores, dentre eles: idade, sexo, utilização de dispositivos invasivos, alteraçôes do sistema imune, maior gravidade do AVC, níveis mais baixos de consciência, presença de disfagia, presença de incontinência urinária e tempo de internação prolongado ${ }^{13,14}$.

No entanto, pesquisas têm demonstrado que o cuidado do paciente com AVC agudo, quando realizado por uma equipe profissional treinada, está associado à diminuição das taxas de complicaçôes no período de internação, dentre elas as ITUs e pneumonia ${ }^{14,15}$.

A segunda complicação mais prevalente neste estudo foram as úlceras por pressão, em estágios II e III. Ressalta-se que possivelmente exista a subnotificação das úlceras por pressão em estágio I, por parte dos profissionais da saúde. Isso limita a comparação com outros estudos e pode ter contribuído também na hierarquização das complicações apresentadas, podendo assumir o primeiro lugar.

As alteraçóes na mobilidade experimentadas por vários pacientes pós-AVC aumentam o risco de desenvolverem esse comprometimento. Além disso, outros fatores podem favorecer o aparecimento das úlceras por pressão, tais como: idade, doenças crônicas, medicamentos, nutrição, umidade, higiene e outros ${ }^{12}$. Devemse considerar as úlceras por pressão como um problema de saúde que acarreta importantes repercussôes, tanto para o paciente quanto para a família e o sistema público, uma vez que os pacientes apresentam diminuição da qualidade de vida e aumentam o risco e infecçôes que inclusive podem levá-los à morte. Ainda, no que diz respeito ao sistema público, as úlceras por pressão acarretam um consumo importante de recursos, um aumento considerável de dias de internação hospitalar e cada vez mais demandas de cuidados ${ }^{12}$.

Outro dado interessante evidenciado pelo estudo foi a baixa ocorrência de flebite, apesar do alto número de pacientes que utilizaram acesso venoso periférico. Esse fato reflete a qualidade da assistência, no que diz respeito aos cuidados dispensados na prevençáo desse tipo de complicação, especialmente pela equipe de enfermagem, que está diretamente envolvida com esses cuidados.

Corroborando estudos citados anteriormente os resultados do modelo de regressão logística evidenciaram que as variáveis idade, tempo de internação e uso de dispositivos invasivos foram associadas a uma maior ocorrência de complicaçóes.

Como não foi possível coletar os dados referentes à gravidade do paciente, essa variável não participou do modelo de regressão logística. Entretanto, é amplamente discutido na literatura que esse fator está associado com a presença de complicaçóes ${ }^{13,15}$.

\section{Conclusão}

Os fatores que se associaram significativamente com a presença de complicaçóes foram: idade, tempo de internação e uso de dispositivos invasivos. Dentre as complicaçóes, a ITU, as úlceras por pressão e a pneumonia foram as mais prevalentes nos pacientes durante a internação. Identificou-se que a média de permanência foi de 11 dias.

Uma das limitações apresentadas pelo estudo é que os dados foram coletados dos prontuários e, nesse sentido, as informaçóes foram condicionadas ao registro feito pelos profissionais. Dessa forma, o estudo não pôde analisar a associação entre a gravidade do paciente e a 
presença de complicaçóes, embora na literatura já esteja bem descrita essa relação.

Este estudo reforça a necessidade de atuação multiprofissional direcionada à diminuição do tempo de internação, por meio da mobilização precoce desses pacientes. Além disso, aponta-se também para a importância do estabelecimento de critérios no uso de dispositivos invasivos.

Recomenda-se o desenvolvimento de outros trabalhos, contemplando os tipos de dispositivos associados com as complicaçóes nesses pacientes, o que poderá desvelar situações não percebidas neste trabalho.

\section{Referências}

1. Organização Mundial da Saúde. Manual STEPS de Acidentes Vascular Cerebrais da OMS: enfoque passo a passo para a vigilância de acidentes vascular cerebrais [internet]. Genebra: OMS; 2006 [acesso em 2013 ago 12]. Disponível em: http://pt.scribd.com/doc/46616252/Avc-Livro-Oms

2. Ministério da Saúde. Portaria 664, de 12 de abril de 2012. Aprova o Protocolo Clínico e Diretrizes Terapêuticas Trombólise no acidente vascular cerebral isquêmico agudo [internet]. Brasília: Ministério da Saúde; 2012 [acesso em 2013 ago 16]. Disponível em: ftp://ftp.saude.sp.gov. br/ftpsessp/bibliote/informe_eletronico/2012/iels.abr.12/ Iels71/U_PT-MS-GM-664_120412.pdf

3. Rolim CLRC, Martins M. Qualidade do cuidado ao acidente vascular cerebral isquêmico no SUS. Cad saúde pública [periódico na internet]. 2011 [acesso em 2013 jul 19];27(11):2106-16. Disponível em: http://www.scielosp. $\mathrm{org} / \mathrm{pdf} / \mathrm{csp} / \mathrm{v} 27 \mathrm{n} 11 / 04 . \mathrm{pdf}$

4. Smeltzer SC, Bare BG. Brunner \& Suddarth/ Tratado de Enfermagem médico-cirúrgica. 12. ed. Rio de Janeiro: Guanabara Koogan; 2012.

5. Gagliardi RJ, Raffin CN, Fábio SRC. Tratamento da fase aguda do acidente vascular cerebral. Projeto Diretrizes. [internet] 2001 [acesso em 2013 ago 12]. Disponível em: http://www. projetodiretrizes.org.br/projeto_diretrizes/010.pdf

6. Paulo RB, Guimarães TM, Helito PVP, Marchiori PE, Yamamoro FI, Mansur LL, et al. Acidente vascular cerebral isquêmico em uma enfermaria de neurologia: complicaçôes e tempo de internação. Rev Assoc Med Bras [internet]. 2009 [acesso em 2013 abr 25];55(3):313-6. Disponível em: http://www.scielo.br/pdf/ramb/v55n3/v55n3a25.pdf

7. Ministério da Saúde. Sexo e idade dos pacientes vítimas de AVC no Brasil, no ano de 2011 [internet]. Brasília: Datasus; [acesso em 2013 ago 22]. Disponível em: http://www2.datasus.gov.br/DATASUS/index.php?area $=02$

8. Oliveira MPF, Novaes MRCG. Perfil socioeconômico, epidemiológico e farmacoterapêutico de idosos institucionalizados em Brasília, Brasil. Cienc saúde coletiva [periódico na internet]. 2013 [acesso em 2014 jul 19];18(4):1069-78. Disponível em: http://www.scielo.br/pdf/csc/v18n4/20.pdf

9. Faria CM, Abudab D, Rodrigues CL, Gorios C, Armond JE, Bertagnon JRD, et al. Perfil epidemiológico dos idosos incluídos no programa acompanhante de idosos (PAI). Arq Geriatr Gerontol [periódico na internet]. 2013 [acesso em 2015 maio 25];7(4):253-58. Disponível em: http://sbgg. org.br/publicacoes-cientificas/revista-geriatria-gerontologia

10. Leite HR, Nunes APN, Corrêa CC. Perfil epidemiológico e qualidade de vida dos pacientes acometidos por acidente vascular encefálico. Arq Ciênc Saúde da UNIPAR [periódico na internet]. 2011 [acesso em 2015 ago 10];15(1):15-21. Disponível em: http://revistas.unipar.br/saude/article/view/3687/2389

11. Costa VSP, Guimarães PSR, Fernandes KBP, Probst VS, Marques AS, Fujisawa DS. Prevalência de fatores de risco para ocorrência de acidente vascular encefálico em idosos. Fisioter mov [periódico na internet]. 2014 [acesso em 2015 ago. 17];7(4):555-63. Disponível em: http://www.scielo.br/scielo. php?script=sci_pdf\&pid=S0103-51502014000400555\&lng $=$ en\&nrm=iso\&tlng=en

12. Moreno CL, Salinas MP, Pappalardo FR, Rodríguez LL. Prevención de úlceras por presión y lesiones musculoesqueléticas: paciente con ictus. Gerokomos [periódico na internet]. 2012 [acesso em 2013 nov 5];23(1):42-6. Disponível em: http://scielo.isciii.es/pdf/geroko/v23n1/helcos3.pdf

13. Westendorp WF, Nederkoorn PJ, Vermeij J, Dijkgraaf MG, Beek DV. Post-stroke infection: A systematic review and meta-analysis. BMC neurol online [serial online]. 2011 Set [cited 2015 Sep 20];11-110. Disponível em: http://www. biomedcentral.com/content/pdf/1471-2377-11-110.pdf

14. Moreira T, Grujic J, Michel P. Early mobilization out of bed after ischaemic stroke reduces severe complications bub not cerebral blood flow: a randomized controlled pilot trial. Clin rehabil [periódico on-line]. 2012 May [acesso em 10 set. 2015];26(5)451-9. Disponível em: http://web.b.ebscohost.com/ehost/pdfviewer/ pdfviewer?vid=3\&sid=fb43d694-dfdd-4224-89cf -d0def7e6a12b\%40sessionmgr111\&hid=113

15. Ingeman A, Andersen G, Hundborg HH, Svendsen ML, Johnsen SP. Processes of care and medical complications in patients with stroke. Stroke [periódico on-line] 2011 Jan [acesso em 2015 ago. 17];42:167-172. Disponível em: http://stroke.ahajournals.org/content/42/1/167.long

\section{Como citar este artigo:}

Melo LS, Emerick LMS, Alves PNM, Rocha TB, Gouveia VR, Guimarães GL, et al. Acidente vascular cerebral: achados clínicos e principais complicaçóes. Rev. Aten. Saúde. 2016;14(48):48-53. 